\title{
Generalized solution of a nonlinear optimal control of the heel angle of a rocket
}

\author{
Touffik Bouremani ${ }^{1}$ and Djamel Benterki ${ }^{2}$ \\ ${ }^{1}$ Laboratory of applied Mathematics, LAMA, Faculty of Technology, Setif-1 Ferhat Abbas University, 19000, Algeria. \\ ${ }^{2}$ Laboratory of Fundamental and Numerical Mathematics LMFN, Department of Mathematics, Faculty of Sciences, Setif-1 Ferhat \\ Abbas University, 19000, Algeria.
}

Received: 13 June 2021, Accepted: 16 June 2021

Published online: 9 July 2021.

\begin{abstract}
We apply the User's Guide on Dynamics Programming described in [2] to obtain a rigorous and theoretically justified solution of the optimal control problem formulated in [3] as an unsolved problem, and studied in [1] using Pontryagin's Maximum Principle. We use a certain refinement of Cauchy's method of characteristics for stratified Hamilton-Jacobi equations to describe a large set of admissible trajectories and to identify a domain on which the value function exists and is generated by a certain admissible control and, its optimality is justified by the use of one of the well-known verification theorems as an argument for sufficient optimality conditions.
\end{abstract}

Keywords: Optimal control, differential inclusion, Pontryagin's maximum principle, dynamic programming, Hamiltonian flow.

\section{Introduction}

The aim of this paper is to apply step by step the dynamic programming theoretical algorithm, described in [2] to obtain a more rigorous and complete theoretically justified solution of the problem formulated as Example 7.3.17 in [3] as an unsolved problem, and studied partially in [1].

\section{Position of the problem}

The goal of the work [1] is to bring the rocket with a constant mass $m$ to an orbit altitude chosen in advance with a maximum lateral offset. Here, the control represents the heel angle of the rocket. The problem is therefore to determine the optimal trajectory of this rocket. This leads us to solve the optimal control problem of minimizing the cost functional:

$$
\begin{cases}\min \mathscr{C}(u, T)=-x_{1}(T), & \\ x_{1}^{\prime}=v_{1}, & x_{1}(0)=0, \\ x_{2}^{\prime}=v_{2}, & x_{2}(0)=0, \\ v_{1}^{\prime}=\frac{a}{m} \cos (u(t)), & v_{1}(0)=0, \\ v_{2}^{\prime}=\frac{a}{m} \sin (u(t))-g, & v_{2}(0)=0, \\ x_{2}(T)=h, v_{1}(T)=v_{c}, v_{2}(T)=0, & \\ u \in \mathbb{R}, t \in[0, T], T \text { free, } & \end{cases}
$$

\footnotetext{
* Corresponding author e-mail: ${ }^{1}$ touffik.bouremani@ univ-setif.dz and ${ }^{2}$ djbenterki@ univ-setif.dz
} 
such that, $x(t)=\left(x_{1}(t), x_{2}(t)\right)$ : the position of the rocket; $v(t)=\left(v_{1}(t), v_{2}(t)\right)$ : his speed; $u(t)$ : the heel angle of the rocket (which is actually the control function); $a>0$ : is a positive real number representing the Thrust force module; $g$ : the gravitational acceleration.

\section{The dynamic programming formulation}

problem 1 Given $T, \alpha>0$, find:

$$
\inf _{u(.)} \mathscr{C}(y, u(.)), \forall y \in Y_{0}
$$

subject to:

$$
\begin{aligned}
& \mathscr{C}(y, u(.))=G(x(T))+\int_{0}^{T} f_{0}(x(t), u(t)) d t, T \text { free, } \\
& x^{\prime}(t)=f(x(t), u(t)), u(t) \in U(x(t)) \text { a.e. }([0, T]), x(0)=y, \\
& x(t) \in Y_{0}, \forall t \in[0, T), x(T) \in Y_{1} \\
& x(.)=\left(x_{1}(.), x_{2}(.), x_{3}(.), x_{4}(.)\right)=\left(x_{1}(.), x_{2}(.), v_{1}(.), v_{2}(.)\right),
\end{aligned}
$$

defined by the following data:

$$
\begin{aligned}
& f(x, u)=\left(x_{3}, x_{4}, \frac{a}{m} \cos u(t), \frac{a}{m} \sin u(t)-g\right), f_{0}(x, u)=0, \\
& U(x)=U=\mathbb{R}, G(\xi)=-\xi_{1}, \forall \xi=\left(\xi_{1}, \xi_{2}, \xi_{3}\right) \in Y_{1} . \\
& Y_{0}=\mathbb{R}_{+}^{4}, Y_{1}=\mathbb{R}_{+}^{*} \times\left\{\left(h, v_{c}, 0\right)\right\} .
\end{aligned}
$$

\subsection{Characterization of the Hamiltonian}

The first step of the Dynamic Programming Approach consists in characterization of the true Hamiltonian of the problem. The pseudo-Hamiltonian $\mathscr{H}(x, p, u)=<p, f(x, u)>+f_{0}(x, u)$ is given in our case by:

$$
\begin{aligned}
& \mathscr{H}(x, p, u)=p_{1} x_{3}+p_{2} x_{4}-g p_{4}+\frac{a}{m} \phi(u), \\
& \phi(u)=p_{3} \cos u+p_{4} \sin u .
\end{aligned}
$$

The Hamiltonian and the corresponding multifunction of minimum points are given by the formulas:

$$
\begin{aligned}
H(x, p) & =\min _{u \in U} \mathscr{H}(x, p, u)=p_{1} x_{3}+p_{2} x_{4}-g p_{4}+\frac{a}{m} \min _{u \in U} \phi(u), \\
\widehat{U}(x, p) & =\{u \in U ; \mathscr{H}(x, p, u)=H(x, p)\}
\end{aligned}
$$

Therefore, the Hamiltonian function as well as the corresponding multifunction of minimum points turn out to be defined on $Z$ by:

$$
\begin{aligned}
& H(x, p)=p_{1} x_{3}+p_{2} x_{4}-g p_{4}+\frac{a}{m} \phi(\widehat{u}(p)),(x, p) \in Z \\
& \widehat{u}(p)=\left\{\begin{array}{l}
\arctan \frac{p_{4}}{p_{3}}, \text { if }\left\{\begin{array}{l}
p_{3}<0, p_{4}<0 \\
p_{3}+p_{4}<0, p_{3}>0 \\
p_{3}+p_{4}<0, p_{4}>0
\end{array}\right. \\
\frac{\pi}{2}, \quad \text { if } p_{3}=0, p_{4}<0 \\
\frac{3 \pi}{2}, \quad \text { if } p_{3}=0, p_{4}>0
\end{array}\right.
\end{aligned}
$$

First, we remark that the Hamiltonian $H(.,$.$) as well as its domain Z$ are $\mathscr{C}^{1}$-stratified by the stratification $S_{H}=\left\{Z_{-,-}\right.$, $\left.Z_{+,-}, Z_{-,+}, Z_{0, \pm}\right\}$ defined by:

$$
\begin{aligned}
& Z_{-,-}=\left\{(x, p) \in Z ; p_{3}<0, p_{4}<0\right\} \\
& Z_{+,-}=\left\{(x, p) \in Z ; p_{3}+p_{4}<0, p_{3}>0\right\} \\
& Z_{-,+}=\left\{(x, p) \in Z ; p_{3}+p_{4}<0, p_{4}>0\right\} \\
& Z_{0, \pm}=\left\{(x, p) \in Z ; p_{3}=0, p_{4} \in \mathbb{R}^{ \pm}\right\} .
\end{aligned}
$$




\section{Set of terminal transversality points}

Next, we need to compute the set of terminal transversality values defined in the general case by:

$$
Z^{*}=\left\{(\xi, q) \in Y_{1} \times \mathbb{R}^{4}, H(\xi, q)=0,<q, \bar{\xi}>=D G(\xi) \bar{\xi} \forall \bar{\xi} \in T_{\xi} Y_{1}\right\}
$$

Lemma 1. The set of terminal transversality values $Z^{*}$ in our case is given by the formulas:

$$
\begin{aligned}
& Z^{*}=Z_{-,-}^{*} \cup Z_{+,-}^{*} \cup Z_{0,-}^{*} \\
& Z_{-,-}^{*}=\left\{\left(\left(\xi_{1}, h,-\frac{a}{m} \sqrt{q_{3}^{2}+q_{4}^{2}}-g q_{4}, 0\right),\left(-1, q_{2}, q_{3}, q_{4}\right)\right)\right. \text {; } \\
& \left.\xi_{1}>0, q_{2} \in \mathbb{R}, q_{3}<0, q_{4}<\frac{a}{\sqrt{m^{2} g^{2}-a^{2}}} q_{3}\right\} \\
& Z_{+,-}^{*}=\left\{\left(\left(\xi_{1}, h, \frac{a}{m} \frac{q_{3}^{2}-q_{4}^{2}}{\sqrt{q_{3}^{2}+q_{4}^{2}}}-g q_{4}, 0\right),\left(-1, q_{2}, q_{3}, q_{4}\right)\right)\right. \text {; } \\
& \left.\xi_{1}>0, q_{2} \in \mathbb{R}, q_{3}+q_{4}<0, q_{3}>0\right\} \\
& Z_{0,-}^{*}=\left\{\left(\xi_{1}, h,\left(\frac{a}{m}-g\right) q_{4}, 0\right),\left(-1, q_{2}, 0, q_{4}\right) ; \xi_{1}>0, q_{2} \in \mathbb{R}, q_{4}<0\right\}
\end{aligned}
$$

\section{Generalized Hamiltonian and characteristic flow}

The first main computational operation consists in the backward integration (for $t \leq 0$ ), of the Hamiltonian inclusion:

$$
\left(x^{\prime}, p^{\prime}\right) \in d_{S}^{\#} H(x, p),(x(0), p(0))=z=(\xi, q) \in Z^{*},
$$

defined by the generalized Hamiltonian field $d_{S}^{\#} H(.,$.$) :$

$$
\begin{aligned}
& d_{S}^{\#} H(x, p)=\left\{\left(x^{\prime}, p^{\prime}\right) \in T_{(x, p)} Z ; x^{\prime} \in f(x, \widehat{U}(x, p)),\right. \\
& \left.<x^{\prime}, \bar{p}>-<p^{\prime}, \bar{x}>=D H(x, p)(\bar{x}, \bar{p}), \forall(\bar{x}, \bar{p}) \in T_{(x, p)} Z\right\}
\end{aligned}
$$

As it is specified in the algorithm given in [2], for each terminal point $z=(\xi, q) \in Z_{1}^{*}$ one should identify the maximal solutions: $X^{*}()=.(X(),. P()):. I(z)=\left(t^{-}(z), 0\right] \rightarrow Z$, of the Hamiltonian inclusion satisfy the following conditions:

$$
\begin{aligned}
& X(t) \in Y_{0}, \forall t \in I_{0}(z)=\left(t^{-}(z), 0\right) \\
& H(X(t), P(t))=0, \forall t \in I(z) \\
& X^{\prime}(t)=f(X(t), u(t)), u(t) \in \widehat{U}\left(X^{*}(t)\right) \text { a.e. } I_{0}(z) .
\end{aligned}
$$

Since the manifolds $Z_{-,-}, Z_{+,-} \subset Z$, are open subsets, one has:

$$
\begin{aligned}
& d_{S}^{\#} H_{-,-}(x, p)=\left\{\left(\frac{\partial H_{-,-}}{\partial p}(x, p),-\frac{\partial H_{-,-}}{\partial x}(x, p)\right)\right\},(x, p) \in Z_{-,-} \\
& d_{S}^{\#} H_{+,-}(x, p)=\left\{\left(\frac{\partial H_{+,-}}{\partial p}(x, p),-\frac{\partial H_{+,-}}{\partial x}(x, p)\right)\right\},(x, p) \in Z_{+,-} .
\end{aligned}
$$

The Hamiltonian system on the stratum $Z_{-,-}$

On the open stratum $Z_{-,-}$for which $p_{3}<0, p_{4}<0$, the differential inclusion coincides with the nonlinear Hamiltonian system:

$$
\left\{\begin{array}{l}
\left(x_{1}^{\prime}, x_{2}^{\prime}, x_{3}^{\prime}, x_{4}^{\prime}\right)=\left(x_{3}, x_{4},-\frac{a}{m} \frac{p_{3}}{\sqrt{p_{3}^{2}+p_{4}^{2}}},-\frac{a}{m} \frac{p_{4}}{\sqrt{p_{3}^{2}+p_{4}^{2}}}-g\right) \\
\left(p_{1}^{\prime}, p_{2}^{\prime}, p_{3}^{\prime}, p_{4}^{\prime}\right)=\left(0,0,-p_{1},-p_{2}\right)
\end{array}\right.
$$


The Hamiltonian system on the stratum $Z_{+,-}$

On the open stratum $Z_{+,-}$for which $p_{3}+p_{4}<0, p_{3}>0$, the differential inclusion coincides with the nonlinear Hamiltonian system:

$$
\left\{\begin{array}{l}
\left(x_{1}^{\prime}, x_{2}^{\prime}, x_{3}^{\prime}, x_{4}^{\prime}\right)=\left(x_{3}, x_{4}, \frac{a}{m} \frac{p_{3}\left[p_{3}^{2}+3 p_{4}^{2}\right]}{\left(p_{3}^{2}+p_{4}^{2}\right)^{\frac{3}{2}}},-\frac{a}{m} \frac{p_{4}\left[p_{4}^{2}+3 p_{3}^{2}\right]}{\left(p_{3}^{2}+p_{4}^{2}\right)^{\frac{3}{2}}}-g\right) \\
\left(p_{1}^{\prime}, p_{2}^{\prime}, p_{3}^{\prime}, p_{4}^{\prime}\right)=\left(0,0,-p_{1},-p_{2}\right) .
\end{array}\right.
$$

\section{The Hamiltonian flow ending on the stratum $Z_{-,-}$}

From the dynamic programming algorithm in [2], it follows that, we must retain only the trajectories $X_{-,-}^{*}(., z), z=(\xi, q) \in Z_{-,-}^{*}$, that satisfy the conditions:

$$
\begin{aligned}
& X_{-,-}^{*}(t, z)=\left(X^{-,-}(t, z), P^{-,-}(t, z)\right) \in Z_{-,-}, \forall t \in\left(\tau^{-,-}(z), 0\right) \\
& H_{-,-}\left(X_{-,-}^{*}(t, z)\right)=0, X^{-,-}(t, z) \in Y_{0},
\end{aligned}
$$

on the maximal intervals $I^{-,-}(z)=\left(\tau^{-,-}(z), 0\right)$, hence the extremity $\tau^{-,-}($.$) is defined by:$

$$
\begin{aligned}
& \tau^{-,-}(z)=\max \left\{\tau_{1}^{-,-}(z), \tau_{2}^{-,-}(z)\right\} \\
& \tau_{1}^{-,-}(z)=\inf \left\{\tau<0 ; P_{3}^{-,-}(t, z)<0, P_{4}^{-,-}(t, z)<0, \forall t \in(\tau, 0)\right\} \\
& \tau_{2}^{-,-}(z)=\inf \left\{\tau<0 ; X^{-,-}(t, z) \in Y_{0}, \forall t \in(\tau, 0)\right\}
\end{aligned}
$$

Further, the second component of the hamiltonian flow $X_{-,-}^{*}(.,$.$) is given by the formulas:$

$$
\begin{aligned}
& P^{-,-}\left(t, q_{2}, q_{3}, q_{4}\right)=\left(-1, q_{2},-t+q_{3},-q_{2} t+q_{4}\right), q_{2} \in \mathbb{R}, q_{3}<0 \\
& q_{4}<\frac{a}{\sqrt{m^{2} g^{2}-a^{2}}} q_{3}
\end{aligned}
$$

Also, the extremity $\tau_{1}^{-,-}($.$) is given by the formulas:$

$$
\tau_{1}^{-,-}\left(q_{2}, q_{3}, q_{4}\right)=\left\{\begin{array}{l}
\max \left\{q_{3}, \frac{q_{4}}{q_{2}}\right\}, \text { if } q_{2}>0, q_{3}<0 \\
q_{3}, \text { if } q_{2} \leq 0, q_{4}<\frac{a}{\sqrt{m^{2} g^{2}-a^{2}}} q_{3}
\end{array}\right.
$$

\section{The Hamiltonian flow ending on the stratum $Z_{+,-}$.}

On the stratum $Z_{+,-}$the maximal interval $I^{+,-}($.$) is of the same form as in above, where the extremity \tau_{1}^{+,-}($.$) is defined$ in this case as:

$$
\tau_{1}^{+,-}(z)=\inf \left\{\tau<0 ; P_{3}^{+,-}(t, z)+P_{4}^{+,-}(t, z)<0, P_{3}^{+,-}(t, z)>0, \forall t \in(\tau, 0)\right\} .
$$

The extremity $\tau_{1}^{+,-}($.$) is given by the formulas:$

$$
\tau_{1}^{+,-}\left(q_{2}, q_{3}, q_{4}\right)= \begin{cases}\frac{q_{3}+q_{4}}{q_{2}+1}, & \text { if } q_{2}>-1, q_{3}+q_{4}<0, q_{3}>0 \\ -\infty & \text { if } q_{2} \leq-1\end{cases}
$$




\subsection{Value function and optimal trajectories}

The natural candidate for value functions and optimal controls in Problem 1 are the extreme ones, defined by the next maximization process:

$$
\begin{aligned}
& W(x)=\left\{\begin{array}{l}
g(x)=-x_{1}, \text { if } x \in Y_{1} \\
W_{0}(x)=\inf _{X(t, b)=x} V(t, b), \text { if } x \in X\left(B_{0}\right) \subset Y_{0}
\end{array}\right. \\
& \widehat{B}(x)=\left\{(t, b) \in B ; X(t, b)=x, V(t, b)=W_{0}(x)\right\}
\end{aligned}
$$

Lemma 2. (1) The mapping $X^{-,-}(., ., ., .,):. B^{-,-} \rightarrow Y_{0}^{-,-}$is a diffeomorphism whose inverse $\widehat{B}^{-,-}($.$) is described by:$

$$
\widehat{B}^{-,-}(x)=\left(\widehat{t}^{-,-}(x), \widehat{\xi}_{1}^{-,-}(x), \widehat{q}_{2}^{-,-}(x), \widehat{q}_{3}^{-,-}(x), \widehat{q}_{4}^{-,-}(x)\right), x \in Y_{0}^{-,-} \text {. }
$$

(2) The mapping $X^{+,-}(., ., ., .,):. B^{+,-} \rightarrow Y_{0}^{+,-}$is a diffeomorphism whose inverse $\widehat{B}^{+,-}($.$) is described by:$

$$
\widehat{B}^{+,-}(x)=\left(\widehat{t}^{+,-}(x), \widehat{\xi}_{1}^{+,-}(x), \widehat{q}_{2}^{+,-}(x), \widehat{q}_{3}^{+,-}(x), \widehat{q}_{4}^{+,-}(x)\right), x \in Y_{0}^{+,-}
$$

The results in the Lemma show that the characteristic flows $C_{-,-}^{*}(.,$.$) and C_{+,-}^{*}(.,$.$) are invertible and define the smooth$ partial proper value function:

$$
W_{0}(x)=\left\{\begin{array}{l}
W_{0}^{-,-}(x)=V\left(\widehat{B}^{-,-}(x)\right)=-\widehat{\xi}_{1}^{-,-}(x), x \in Y_{0}^{-,-} \\
W_{0}^{+,-}(x)=V\left(\widehat{B}^{+,-}(x)\right)=-\widehat{\xi}_{1}^{+,-}(x), x \in Y_{0}^{+,-}
\end{array}\right.
$$

Moreover, it follows that the corresponding admissible controls are given by:

$$
\tilde{u}(x)=\left\{\begin{array}{l}
\widetilde{u}^{-,-}(x)=\arctan \left[\frac{\widehat{q}_{2}^{-,-}(x) \hat{t}^{-,-}(x)-\widehat{q}_{4}^{-,-}(x)}{\widehat{t}^{-,-}(x)-\widehat{q}_{3}^{-,-}(x)}\right], x \in Y_{0}^{-,-} \\
\widetilde{u}^{+,-}(x)=\arctan \left[\frac{\widehat{q}_{2}^{+,-}(x) \hat{t}^{+,-}(x)-\widehat{q}_{4}^{+,-}(x)}{\widehat{t}^{+,-}(x)-\widehat{q}_{3}^{+,-}(x)}\right], x \in Y_{0}^{+,-} .
\end{array}\right.
$$

Theorem 1. The corresponding admissible controls $\widetilde{u}($.$) in (26) are optimal for the restriction on its domain Y_{0}^{-,-} \cup Y_{0}^{+,-}$.

\section{Competing interests}

The authors declare that they have no competing interests.

\section{Authors' contributions}

All authors have contributed to all parts of the article. All authors read and approved the final manuscript.

\section{References}

[1] M. Aliane, N. Moussouni, M. Bentobache, Nonlinear optimal control of the heel angle of a rocket, 6th International Conference on Control, Decision and Information Technologies (CoDIT'19) Paris, France / April 23-26, 2019.

[2] Şt. Mirică, User's Guide on Dynamic Programming for autonomous differential games and optimal control problems. Rev. Roumaine Math. Pures Appl. 49(2004), No. 5-6, 501-529.

[3] E. Trélat, Contrôle optimal: théorie et applications. Mathématiques concrètes, Vuibert, Paris (2005). 